\title{
A Large Frequency Ratio Dual-band Microstrip Antenna with Consistent Radiation Pattern for Internet of Sea Applications
}

\author{
Hanguang Liao ${ }^{1}$, Rana Muhammad Bilal ${ }^{1}$, Atif Shamim ${ }^{1}$ \\ ${ }^{1}$ Electrical and Computer Engineering Program, King Abdullah University of Science and Technology, Thuwal, Saudi Arabia \\ E-mail address: Hanguang.liao@kaust.edu.sa Atif.shamim@kaust.edu.sa
}

\begin{abstract}
Sensing and retrieving data from ocean to land are challenging tasks, while the Internet of Sea concept provides a realistic solution to that. A large frequency ratio dual-band microstrip antenna design working at GSM, LoRa, and BLE bands with consistent radiation pattern is proposed for Internet of Sea applications. The antenna is based on a modified Split Ring Antenna which provides two radiating modes, where the frequency ratio is close to 3 . The proposed microstrip antenna is optimized for a better radiation efficiency and consistent radiation pattern at both bands. The method to control the H-plane HPBW of the proposed antenna is provided. The antenna prototype is fabricated and measured, which shows the radiation pattern at both bands are consistent and the H-plane HPBWs are $94^{\circ}$ and $78^{\circ}$, at the lower and higher bands respectively.
\end{abstract}

Index Terms - microstrip antennas, dual-band, large frequcny-ratio, HPBW control, stable radiation pattern.

\section{INTRODUCTION}

Ocean environment has a huge potential for humans to study and explore. To better protect the ocean environment and marine animals, sensing the ocean parameters (like pressure, temperature, concentrations of certain pollutants, etc.) and animal parameters (like body temperature, motions, etc.) are essential. However, transferring the data from the sensor nodes to the researchers has been a challenging task. The commercially available marine sensing systems are expensive and bulky, such as hundreds of kilometers of cables or optical fiber with sensors along the seabed to shore [1]. The concept of Internet of Sea leads us to build sensing and communication systems to solve the problems. We have previously proposed an Internet of Sea system containing a flexible animal tag design [2-3] and a small floating receiver design [4] for small area monitoring. In that system, sensors' data are temporarily stored in the animal tag and offloaded to nearby floating receivers when marine animals breathe on sea surface. However, the floating receiver in [4] is only applicable for small area monitoring, covering an area with a radius of around 240 meters, which is not sufficient for large area monitoring for hundreds of $\mathrm{km}^{2}$. We have designed a new buoy system with multiple communication abilities including Extended Global System for Mobile communications (EGSM-900), Long Range (LoRa, 902 to $928 \mathrm{MHz}$ ), and

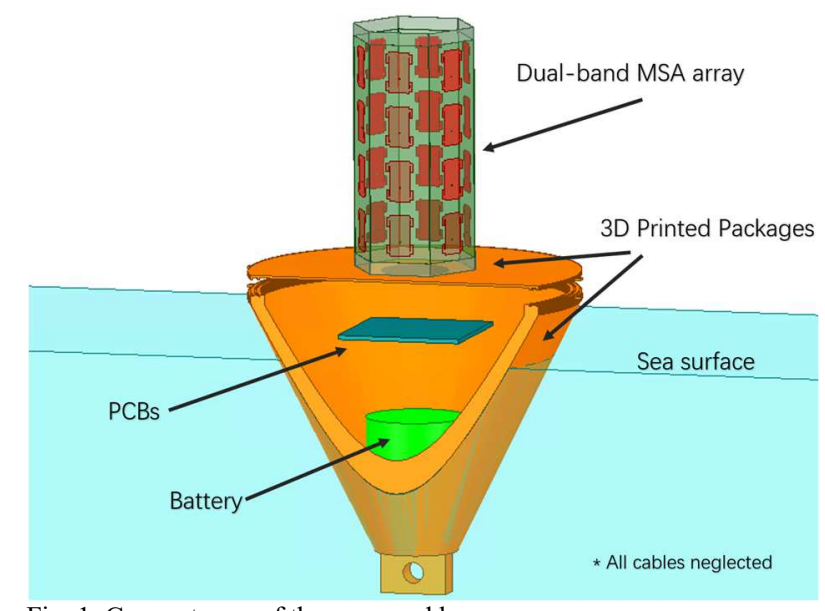

Fig. 1. Concept map of the proposed buoy.

Bluetooth Low Energy (BLE, 2.4 to $2.48 \mathrm{GHz}$ ) for large area monitoring.

The proposed buoy system structure is shown in Fig. 1. The missions of buoys are to detect animal tags and retrieve data from them when animal tags float up to the sea surface after collecting enough data, where the animal tag design is similar to that in [2]. As can be seen from Fig. 1 the proposed buoy contains dual-band antenna arrays on top, essential PCBs inside, and batteries at the bottom. We will focus on the dual-band antenna design in this paper. Microstrip Antenna (MSA) arrays are widely used due to the easy implementation and low-cost characteristics. There are some special requirements for our dual-band MSA. The polarization of the dual-band antenna should be vertical at both bands to be consistent with the GSM base-stations at coasts and our animal tag BLE antennas (vertical polarization). Besides, the Half Power Beam-Width (HPBW) of H-plane should be close at both bands, since the antenna array on each side should cover the same H-plane angle at both bands to form good overall coverage above sea surface.

From the working requirements described, a dual-band MSA working at both $900 \mathrm{MHz}$ (GSM and LoRa) and $2.4 \mathrm{GHz}$ (BLE), with vertically polarization and consistent radiation pattern at both bands is needed. There are some papers reporting dual-band MSAs which has a FR over 2 [5-11]. 


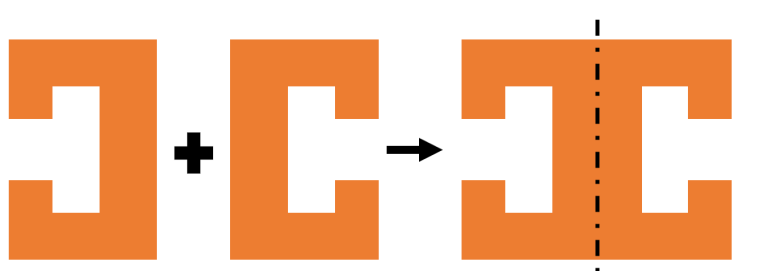

Fig. 2. The evolution of the proposed antenna.

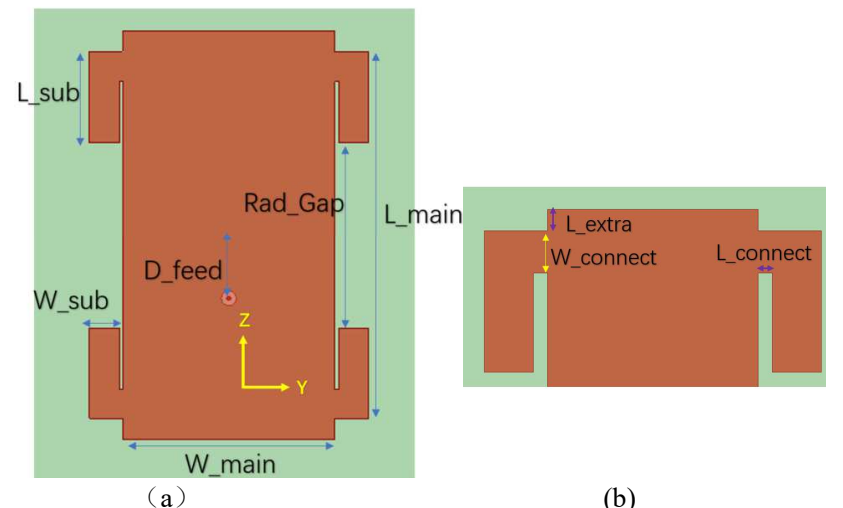

Fig. 3. The geometry of the proposed antenna: (a) planar view; (b) detail view.

Although some of them achieved a larger FR over 4 [5-6], the size of the antenna becomes large [5], or the radiation efficiency is low at lower band (LB) [6]. An interesting approach of realizing dual-band MSA which has FR close to 3 and consistent radiation performance is using the higher mode of microstrip Split Ring Antenna (SRA) [7-11]. In [7], the radiation characteristics are analyzed for the basic and non-optimized SRA structure, which has proven the concept well, but the radiation efficiency at LB is low, as well as the gain. Researchers have improved the SRA for some perspectives [8-11], like FR adjustment [8], miniaturization [9], array configuration [10], and using flexible substrate [11]. However, none of these papers above have investigated the radiation pattern similarity of the SRA at LB and HB.

In this paper, a dual-band antenna working at 0.9 and 2.4 $\mathrm{GHz}$ bands, with stable polarization and radiation pattern at both bands, is proposed. The idea is based on the two separated modes of microstrip SRA which can generate consistent radiation patterns at both bands even if FR is relatively large. The radiation pattern control study, especially the HPBW control method, is provided in this paper. The proposed antenna has a good radiation efficiency at both bands and close HPBW in H-plane, which satisfies all the requirements for the proposed Internet of Sea system.

\section{ANTENNA DESIGN}

The evolution of the proposed antenna is shown in Fig. 2. Two rectangular microstrip SRAs, whose radiating resonant modes have a FR close to 3 [7], are placed back to back, and then are emerged to one MSA. Although each SRA can work individually, the back-to-back rectangular SRA has better cross-polarization suppression due to the symmetric geometry, if the antenna is fed along the center line shown in

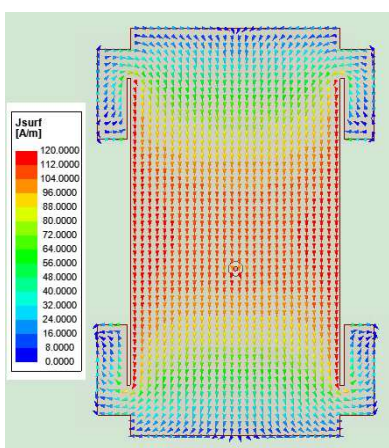

(a)

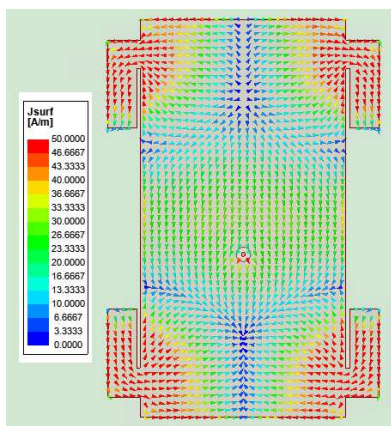

(c)

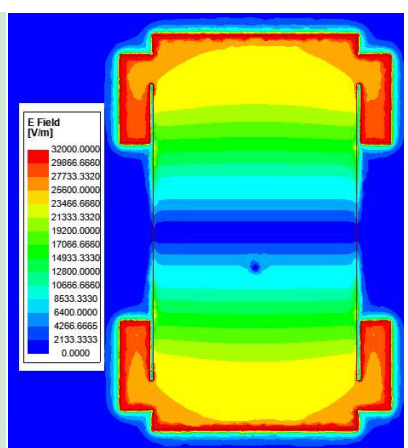

(b)

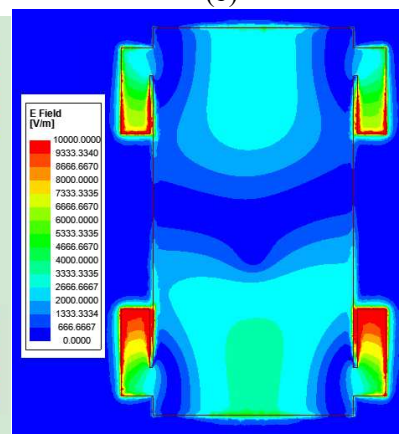

(d)
Fig. 4. Current and E-field distributions of the proposed antenna: (a) current distribution at LB; (b) E-field distribution at LB; (c) current distribution at $\mathrm{HB}$; (d) E-field distribution at $\mathrm{HB}$.

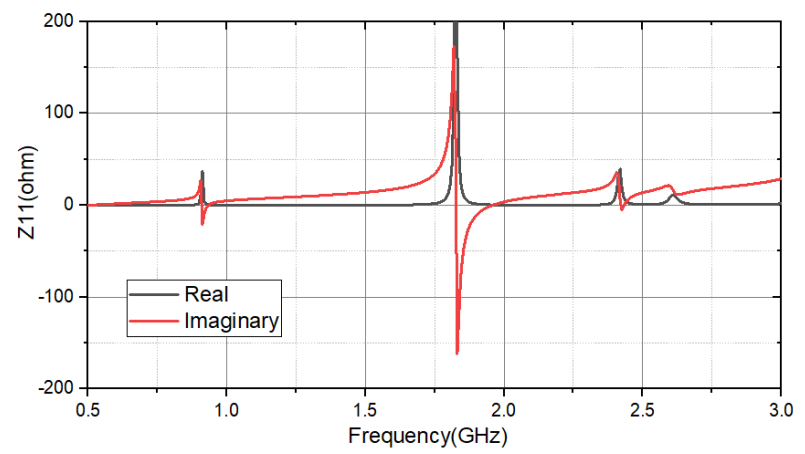

Fig. 5. The simulated input impedance.

Fig. 2. After optimization for radiation efficiency enhancement and radiation pattern control, the geometry of the proposed dual-band MSA is shown in Fig. 3. The antenna is designed on Rogers Laminates RT/duroid 5880 with a dielectric constant of 2.2 and a loss tangent of 0.0004 . The split in traditional SRA is here enlarged as the radiation gap with length Rad_Gap in Fig. 3 (a). To clearly explain the antenna configuration, we call the big rectangular patch in the center as main patch, and it has four sub-patches at each corner which are connected to main patch by four connect arms. An extended length L_extra is added on the main patch on both sides to perform better FR control as shown in Fig. 3 (b). Although not drawn here, there is a square ground plane with each side $150 \mathrm{~mm}$, at the backside of the laminates as this is an MSA. The dimensions of the proposed dual-band MSA are listed here: $\mathrm{L}$ main $=87 \mathrm{~mm}, \mathrm{~W} \_$main $=50 \mathrm{~mm}, \mathrm{~L} \_\mathrm{sub}=21.5$ $\mathrm{mm}, \mathrm{W} \_\mathrm{sub}=7 \mathrm{~mm}, \mathrm{~L} \_$connect $=1 \mathrm{~mm}, \mathrm{~W} \_$connect $=7 \mathrm{~mm}$, D_feed $=8.75 \mathrm{~mm}, L_{-}$extra $=5 \mathrm{~mm}$. 


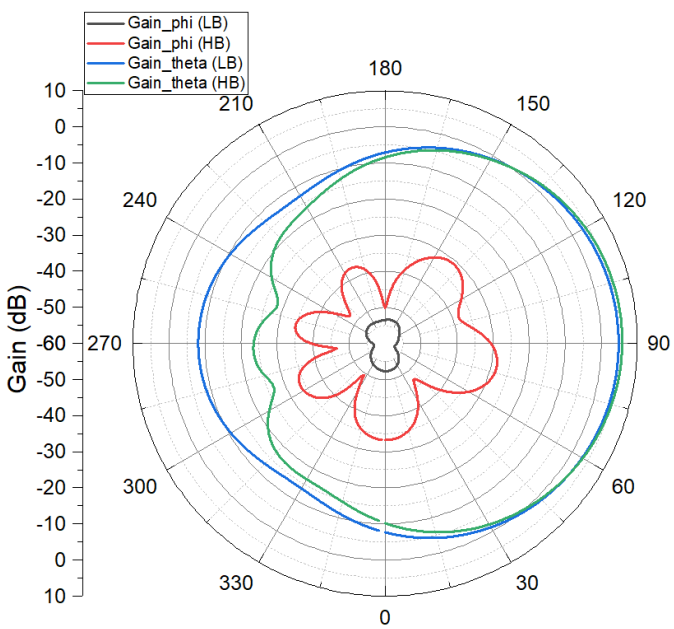

(a)

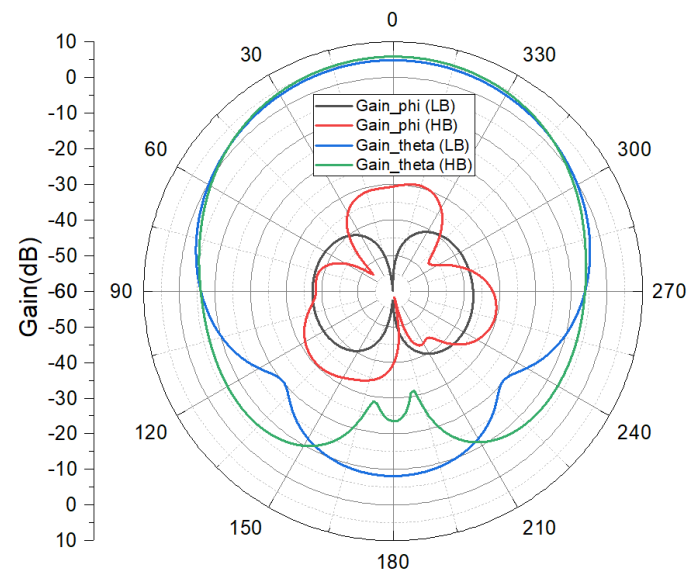

(b)

Fig. 6. The simulated radiation patterns: (a) E-plane; (b) H-plane.

To further verify the working principles of the proposed antenna, we have simulated the antenna by Ansys HFSS. The current density and E-field distribution are shown in Fig. 4. In Fig. 4 ( $a$ and $b$ ), at the LB, we can see that the current distribution on the patch is similar to that of SRA, where we get maximum current at the center and minimum current at the edge of radiation gap. The E-field distribution at LB is also reasonable where we find the maximum E-field is around the radiation gap. The current path from one radiation edge to another one is proximately half guided wavelength, resulting in the LB mode resonates at $0.91 \mathrm{GHz}$ as shown in Fig. 5. The current distribution and E-field densities at $\mathrm{HB}$ mode are shown in Fig. 4 (c and d). Seen from the HB mode, the proposed antenna still has Maximum E-field at the edge of the radiation gap, which is the same as that of LB mode. However, the higher mode has 3 half wavelengths variation from one radiation edge to another one in the current distribution plot. This actually provides us the fundamentals of dual-band MSA which can operate with a $F R=2.7$, close to 3 . This is verified in Fig. 5 that the HB mode is resonant at $2.42 \mathrm{GHz}$. For both lower and higher modes, the radiated wave comes from the same radiation gap, and thus, it keeps the radiation pattern consistent at both bands. Compared to traditional rectangular MSA, the proposed antenna has moved its radiating edges to

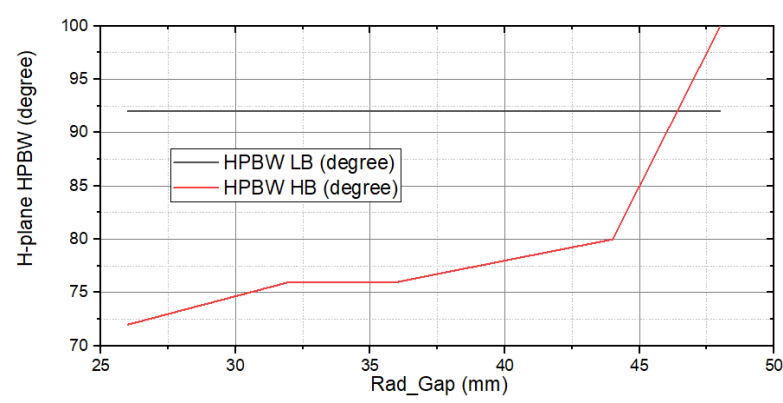

(a)

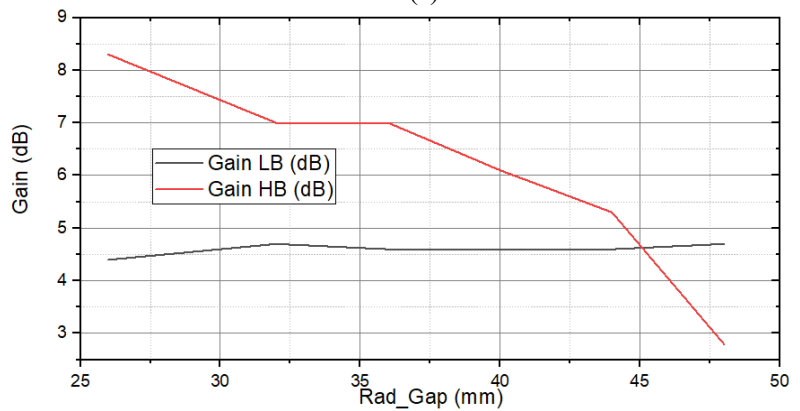

(b)

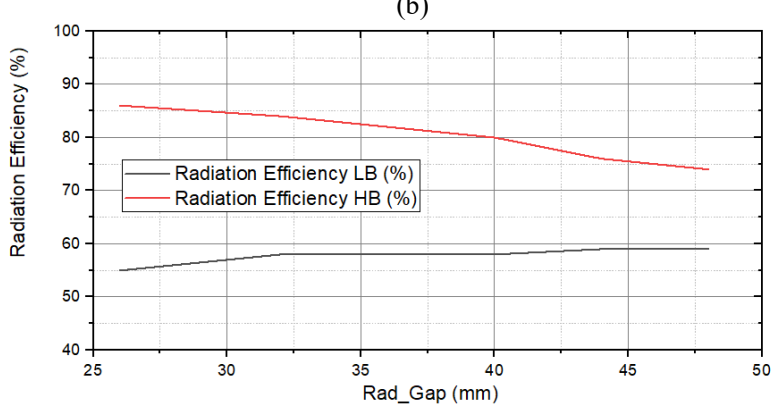

(c)

Fig. 7. Simulations with Rad_Gap: (a) H-plane HPBW; (b) Broadside Gain; (c) Radiation efficiency.

the middle, which will significantly decrease the mutual coupling effect when a greater number of antenna elements are closely packed.

The simulated radiation patterns of the proposed dualband MSA are shown in Fig. 6. The radiation patterns in Eplane at both bands looks identical as can be seen in Fig. 6 (a). It has a co-polarization gain ( $\theta$ component) of $4.7 \mathrm{~dB}$ and 5.3 $\mathrm{dB}$ at $\mathrm{LB}$ and $\mathrm{HB}$, respectively. A very good crosspolarization level is achieved at both bands $(>35 \mathrm{~dB})$ at broadside direction. The H-plane radiation pattern is shown in Fig. 6 (b). The HPBW in H-plane is $92^{\circ}$ and $80^{\circ}$, at $\mathrm{LB}$ and $\mathrm{HB}$ respectively, which are close enough for a dual-band MSA with a FR of 2.7. Thus, we can safely claim that the proposed dual-band MSA has a consistent radiation pattern at both bands.

The method to control the H-plane HPBW, and its effect on gain and radiation efficiency, is investigated. Rad_Gap is important for the radiation performance of the dual-band MSA since the radiated field is coming from the radiation gap to free space. To investigate the effect of the length Rad Gap, we did simulations with various Rad Gap, and the relationships are summarized in Fig.7. Note that by changing Rad_Gap, some other dimensions (L_main, L_sub, W_sub and L_connect) 


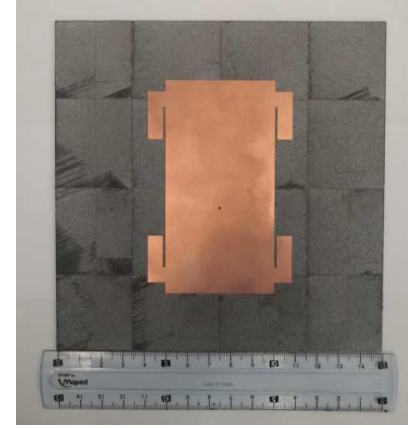

(a)

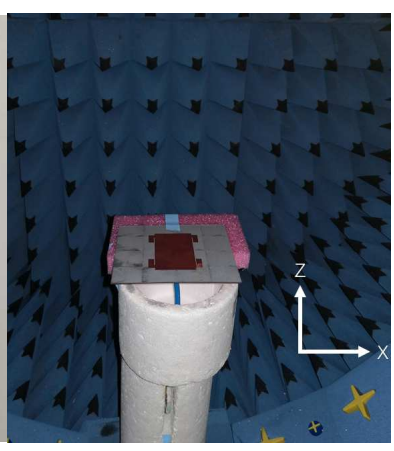

(b)
Fig. 8. Photos: (a) fabricated antenna; (b) test in anechoic chamber.

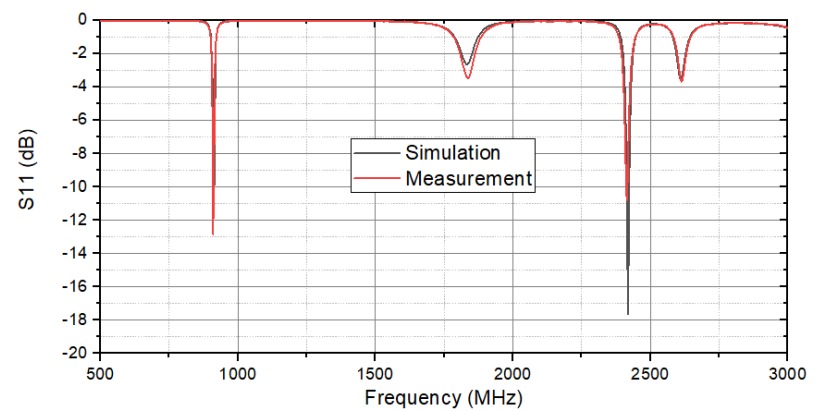

Fig. 9. S parameters.

should also be changed accordingly to make the lower mode and higher mode resonant at 0.9 and $2.4 \mathrm{GHz}$, respectively. Though not shown in this paper, changing those parameters will not affect the radiation performance. The H-plane HPBW is important as we have explained in the introduction section that the H-plane HPBWs at both bands are desired to be close. At $\mathrm{LB}$, all the radiation performances change very slightly because the Rad_Gap is always electrically small $(<0.15 \lambda, \lambda$ is the free space wavelength at LB) as shown in Fig. 7 (a,b,c). However, the higher band radiation performances are sensitive to Rad_gap. As seen from Fig. 7 (a), as the Rad_Gap increases, the HPBW at HB tends to increase. And in Fig. 6 (b), the broadside gain of the MSA at HB decreases slightly as Rad_Gap increases below $44 \mathrm{~mm}$, and decreases significantly as Rad Gap increases above $44 \mathrm{~mm}$. The reason for gain decreasing can be explained by the radiation pattern distortion: when the Rad_Gap is small, the radiation pattern at $\mathrm{HB}$ is a typical broadside radiation pattern which has only one main lobe, similar to that in Fig. 6. Then, as the Rad_Gap increases, the two radiating edges become farther so the combined radiation pattern tends to separate into two grating lobes, causing decreasing in gain, increasing in HPBW and only a small change in radiation efficiency. When Rad_Gap increases to $48 \mathrm{~mm}$, the radiation pattern at HB is no longer a typical broadside radiation but a two-grating-lobe pattern which results in decreasing in broadside gain very significantly. To compromise between a good broadside gain and a close H-plane HPBW, we choose Rad_Gap $=44 \mathrm{~mm}$ in our final design.
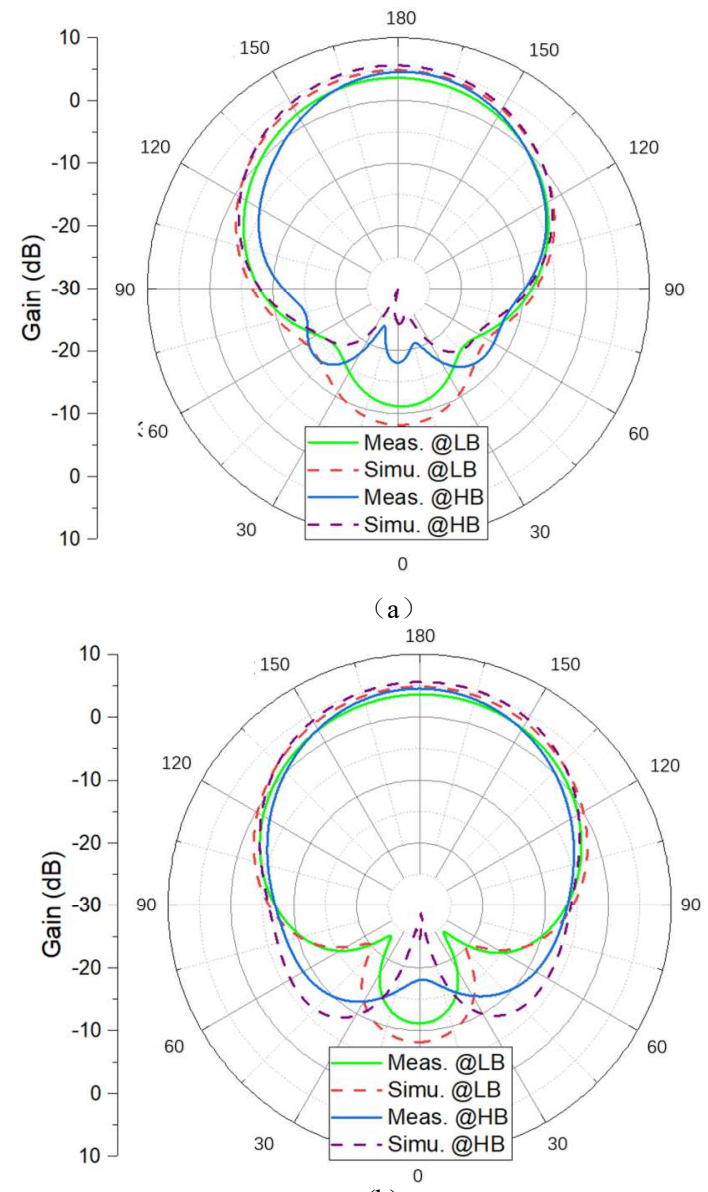

(b)

Fig. 10. Radiation patterns results: (a) E-plane; (b) H-plane.

\section{PROTOTYPING AND MESUREMENT RESULTS}

The proposed antenna is fabricated by a regular milling process using Rogers RT/duroid 5880 laminates. An SMA connector is then soldered on the ground plane side. The fabricated sample is shown in Fig. 8 (a).

The $\mathrm{S}$ parameter of the antenna prototype is measured by Keysight E8363C PNA Network Analyzer, and the result is shown in Fig. 9. From it we can see that antenna is matched at 0.91 and $2.41 \mathrm{GHz}$, with a bandwidth of $6 \mathrm{MHz}$ and $9 \mathrm{MHz}$ respectively. This may be considered as a narrow band but that is the natural physics of small antennas [12] where the patch length is only $0.29 \lambda$ ( $\lambda$ is the free space wavelength at LB).

The radiation pattern is measured by SATIMO Star Lab Anechoic Chamber in Fig. 8 (b) and its results are shown in Fig. 10. For both H-plane and E-plane patterns, the measured results are matched with the simulation result. As can be seen from Fig. 10, the radiation patterns at both bands are controlled to be identical. The measured broadside gain is 3.6 $\mathrm{dB}$ and $4.6 \mathrm{~dB}$ at $\mathrm{LB}$ and $\mathrm{HB}$ respectively. The measured gain is slightly lower than the simulation which may due to the loss from the connector and soldering. The measured H-plane 
TABLE I. COMPARISON BETWEEN SIMILAR WORKS

\begin{tabular}{cccccc}
\hline \hline Reference & $\begin{array}{c}\text { Antenna } \\
\text { Structure }\end{array}$ & Total size $\left(\lambda_{l}{ }^{3}\right)$ & FR & $\begin{array}{c}\text { Gain } \\
(\mathrm{LB} / \mathrm{HB})\end{array}$ & $\begin{array}{r}\text { H-plane HP } \\
(\mathrm{LB} / \mathrm{HB}\end{array}$ \\
\hline$[7]$ & Single layer & $0.84 * 0.84 * 0.008$ & 2.8 & $-1.4 / 5.0$ & NA \\
{$[13]$} & Air substrate & $0.87 * 0.64 * 0.074$ & 1.53 & $8.2 / 7.9$ & NA \\
{$[14]$} & 3D & $0.97 * 0.97 * 0.1$ & 2.1 & $9.4 / 10.6$ & NA \\
{$[15]$} & Single layer & $>0.33 * 0.24 * 0.015$ & 1.8 & $6.5 / 6.8$ & NA \\
This Work & Single layer & $0.45 * 0.45 * 0.005$ & 2.7 & $3.6 / 4.6$ & $94 / 78$ \\
\hline \hline
\end{tabular}

HPBWs are $94^{\circ}$ and $78^{\circ}$, at LB and HB respectively, showing a very small discrepancy with simulation.

To the best of our knowledge, there is no paper in the literature investigating the single layer large FR dual-band microstrip antenna that keeps the consistent radiation pattern on both bands. If we release the requirement of radiation pattern similarity investigation, as they did not show their metrology of the pattern similarity, there are several large FR designs which could be comparable, summarized in Table I [7, 13-15]. The designs in [7, 15] are also single-layer structure which is low profile, robust, and can be easily integrated with circuits, but [7] has a low gain $(-1.4 \mathrm{~dB})$ at LB and [15] only supports the maximum FB of 2 . Although [13, 14] have relatively higher gain than our design, the total size of the antenna, especially the thickness, are much larger than ours. None of those design shows the measured H-plane HPBW to evaluate the radiation pattern similarity.

\section{CONCLUSION AND FUTURE WORKS}

In this paper, a dual-band MSA with relatively large FR and consistent radiation patterns at both bands is proposed. The working mechanism is analyzed by a full-wave simulator, and the HPBW control method is performed. A prototype is then fabricated accordingly, and all the measured results show good consistency between simulation and the practical design. The measured HPBWs are $94^{\circ}$ and $78^{\circ}$, at LB and HB respectively, which is very close and satisfies the requirement of the Internet of Sea applications. The proposed antenna has potentials not only for the Internet of Sea systems, but also for other practical applications where the system is required to operate in a large ratio dual-band mode, such as base stations, IoT nodes and networks. In the future, the array configuration of the proposed antenna and bandwidth enhancement techniques can be further investigated for those practical applications.

\section{REFERENCES}

[1] P. Favali et al., "Seafloor observatory science," in Proc. OceanObs'09: Sustained Ocean Observ. Inf. Soc. (Vol. 2), Venice, Italy, Sep. 2009, doi: 10.5270/OceanObs09.cwp.28.

[2] M. A. Karimi, Q. Zhang, Y. Kuo, S. F. Shaikh, A. Kaidarova, N. Geraldi, M. Hussain, J. Kosel, C. Duarte, A. Shamim, "Flexible tag design for semi-continuous wireless data acquisition from marine animals," in Flexible and Printed Electronic vol. 4, pp. 035006, Sept. 2019, doi: 10.1088/2058-8585/ab423f

[3] Q. Zhang, Y. Kuo, N. Mishra and A. Shamim, "Flexible QuasiIsotropic Antenna for Marine Animals Tagging application," 2018 International Flexible Electronics Technology Conference (IFETC), Ottawa, ON, 2018, pp. 1-2, doi: 10.1109/IFETC.2018.8583987..
[4] H. Liao, Q. Zhang, M. A. Karimi, Y. Kuo, N. Mishra and A. Shamim, "An Additively Manufactured 3-D Antenna-in-Package With QuasiIsotropic Radiation for Marine Animals Monitoring System," in IEEE Antennas and Wireless Propagation Letters, vol. 18, no. 11, pp. 23842388, Nov. 2019, doi: 10.1109/LAWP.2019.2937507.

[5] P. Li, K. M. Luk and K. L. Lau, "A dual-feed dual-band L-probe patch antenna," in IEEE Transactions on Antennas and Propagation, vol. 53, no. 7, pp. 2321-2323, July 2005, doi: 10.1109/TAP.2005.850761.

[6] Shan-Cheng Pan and Kin-Lu Wong, "Dual-frequency triangular microstrip antenna with a shorting pin," in IEEE Transactions on Antennas and Propagation, vol. 45, no. 12, pp. 1889-1891, Dec. 1997, doi: 10.1109/8.650213.

[7] S. I. Latif and L. Shafai, "Polarization Characteristics of Multiband Loaded Microstrip Annular Ring Antennas," in IEEE Transactions on Antennas and Propagation, vol. 57, no. 9, pp. 2788-2793, Sept. 2009, doi: 10.1109/TAP.2009.2027354.

[8] K. Jhamb, L. Li and K. Rambabu, "Frequency adjustable microstrip annular ring patch antenna with multi-band characteristics," in IET Microwaves, Antennas \& Propagation, vol. 5, no. 12, pp. 1471-1478, 16 September 2011, doi: 10.1049/iet-map.2010.0571.

[9] Başaran, S. C.. "Compact dual-band split-ring antenna for $2.4 / 5.2 \mathrm{GHz}$ WLAN applications." Turkish Journal of Electrical Engineering and Computer Sciences 20 (2012): 347-352.

[10] C. Arora, S.S. Pattnaik, R.N. Baral, "Dual Band Microstrip Patch Antenna Array Loaded with Split Ring Resonators and Via Holes" in International Journal of Electronics and Communications Volume 93, 2018, Pages 253-260, ISSN 1434-8411.

[11] Casula, G. A., et al. (2016). "A Multiband Proximity-Coupled-Fed Flexible Microstrip Antenna for Wireless Systems." International Journal of Antennas and Propagation 2016: 8536058.

[12] L. J. Chu, "Physical limitations on omni-directional antennas," J. Appl. Phys., vol. 19, pp. 1163-1175, Dec. 1948.

[13] W. C. Mok, S. H. Wong, K. M. Luk and K. F. Lee, "Single-Layer Single-Patch Dual-Band and Triple-Band Patch Antennas," in IEEE Transactions on Antennas and Propagation, vol. 61, no. 8, pp. 43414344, Aug. 2013, doi: 10.1109/TAP.2013.2260516.

[14] Y. Wang and Z. Du, "Dual-Polarized Dual-Band Microstrip Antenna With Similar-Shaped Radiation Pattern," in IEEE Transactions on Antennas and Propagation, vol. 63, no. 12, pp. 5923-5928, Dec. 2015, doi: 10.1109/TAP.2015.2487520.

[15] S. Maci, G. Biffi Gentili, P. Piazzesi, and C. Salvador, "Dual-band slotloaded patch antenna," Inst. Elect. Eng. Proc. Microwaves Antennas Propagat., vol. 142, no. 3, 1995. 